\title{
Unilateral lenticular infarcts: radiological and clinical syndromes, aetiology, and prognosis
}

\author{
M Giroud, M Lemesle, G Madinier, Th Billiar, R Dumas
}

\begin{abstract}
Objectives-To analyse the clinical features induced by lenticular infarction found in 20 patients, and to analyse the radiological and clinical correlations.

Methods-Eight women and 12 men, mean age 73 years, were included in this study, which was carried out from 1 January 1994 to 30 November 1996. They were characterised by the onset of a lenticular infarction, shown by CT and MRI. A complete neurological and neurocognitive examination, and photon emission computed tomography (SPECT), were performed in all the patients and there was a long clinical follow up.

Results-Two distinct clinical syndromes were identified corresponding to the two anatomical areas of the lenticular nucleus: behavioural and cognitive disorders were associated with infarcts within the globus pallidus, whereas both motor disorders (dystonia) and cognitive disorders were associated with infarcts within the putamen. Outcome was excellent in all the patients for motor function, but slight cognitive disorders, problems with short term memory, and dysphasia persisted for several months. The size of the lesion did not explain these symptoms. By contrast, the slight reduction in cerebral blood flow found in the adjacent frontotemporal area may explain them by a deafferentation or a diaschisis phenomenon.
\end{abstract}

Conclusion-It is possible to identify the clinical symptoms of a single lesion in the pallidus nucleus and in the putaminal nucleus, in which behavioural, cognitive, and movements disorders are important. After an acute and spectacular onset, outcome is in general excellent. A disease of the small arteries must be involved.

\section{(F Neurol Neurosurg Psychiatry 1997;63:611-615)}

Keywords: infarction: lentiform nucleus: motor disorders: cognitive disorders

A knowledge of the functions of the basal ganglia is important.

The lesions that damage the human brain are rarely restricted to a single anatomical structure. Published case reports of lenticular infarction often include patients whose infarcts were not limited to the lenticular nucleus. ${ }^{1}$
However, modern imaging techniques such as CT and MRI are able to identify lesions restricted to the lenticular nucleus.

We present the clinical findings, the radiological features, the aetiologies, and the long term prognosis of 20 patients with an isolated lenticular infarct established by CT and MRI.

\section{Methods}

We studied all patients with an acute lenticular infarct-diagnosed by both CT and MRIwho were admitted to the neurology department between 1 January 1994 and 30 November 1996 (eight women and 12 men). We excluded patients with previous strokes or with associated infarcts in the internal capsule, the caudate nucleus, or the thalamus. All patients were studied by all of us and they had the same investigations including standard blood tests, ECG, transoesophageal echography (TEE), Doppler ultrasound, CT at day 1 and day 10, MRI at day 7, and SPECT at day 10.

Brain CT and MRI were performed using orbitomeatal horizontal sequences and coronal sequences also for MRI. An ischaemic stroke was defined by a hypodense lesion on CT, a hyposignal on $\mathrm{T} 1$ weighted images, and a hypersignal on T2 weighted images with gadolinium uptake after day 7 on MRI. T1 weighted images were acquired on a 1.5 Tesla Siemens MRI machine, with a short TR (480 ms) and a short TE $(15 \mathrm{~ms})$, and T2 weighted images with a TR of $250 \mathrm{~ms}$ and a TE of $20 \mathrm{~ms}$. On CT, lacunar infarcts present as sharply delineated areas of hypodensity which may be round, with a diameter $<1.5 \mathrm{~cm}$, rectangular, or pyramidal. Lacunes appear on MRI as discrete areas with prolonged T1 (hyposignal) and prolonged T2 (hypersignal) images with a diameter $<1.5 \mathrm{~cm}$ and may be related to clinical symptoms as well as pure motor hemiplegia, pure hemianaesthesia, dysarthria-clumsy syndrome, and ataxic hemiplegia. Perfusion of gadolinium allowed the age of the infarct to be determined. The lack of dilatation of the homolateral ventricule indicated that the lesion was recent.

We made the topographic diagnosis of lenticular infarct on CT and MRI using previously reported templates. ${ }^{23}$

The study of cerebral blood flow was performed by scintigraphy with $\mathrm{Hm}$ PAO labelled with technetium producing a single photon emission computed tomography 


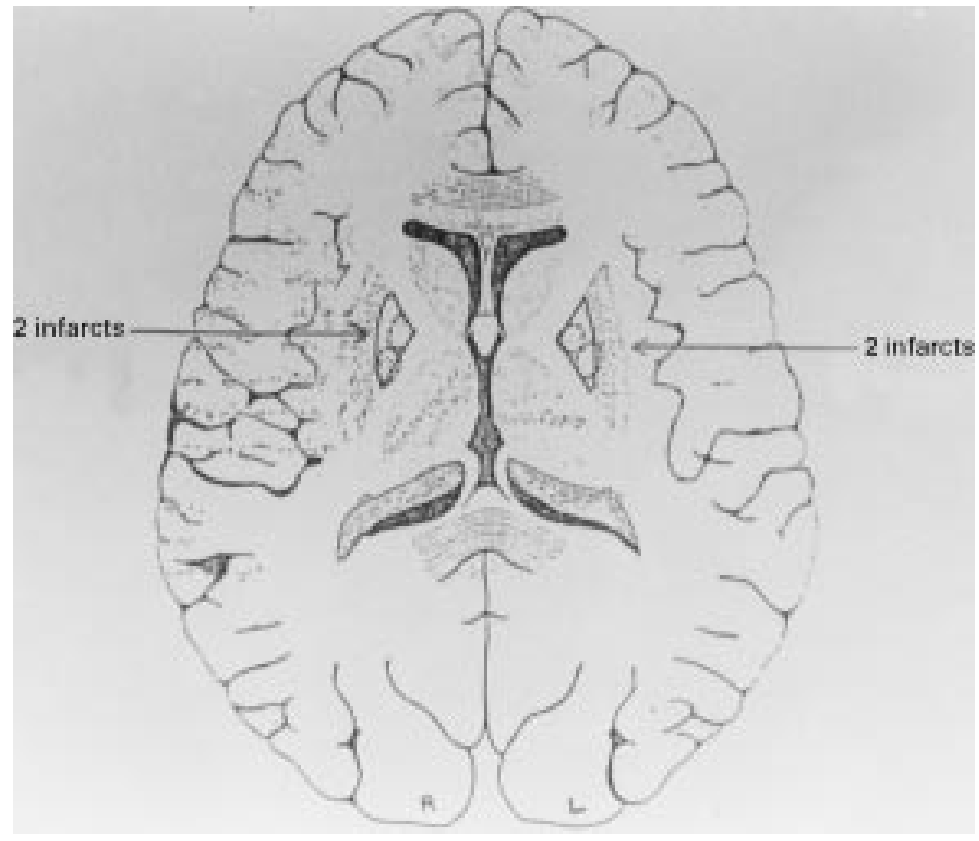

Figure 1 Schematic template showing lesion sites in the four patients with a globus pallidus infarct. on TEE. Presumed lacunar infarction was diagnosed in patients with hypertension ${ }^{9}$ or diabetes, ${ }^{3}$ in the absence of arterial or cardiac sources of emboli.

\section{Results}

There was an infarction within the globus pallidum in four patients and in the putamen in 16. The perfusion of gadolinium disclosed that there were no other infarcts.

GLOBUS PALLIDUM INFARCT (FIG 1)

Table 1 summarises the main characteristics of this infarct. Mean age was 72.5 years. There were three right handed men and one right handed woman. There were two right pallidal infarcts and two left pallidal infarcts. The main symptoms were behavioural with acute abulia defined by as loss of drive, apathy, loss of spontaneous motor activity, loss of emotional affective expression, and reduction of spontaneous thought content and initiative in patients 1 and 2 (left pallidal infarction in both), acute desinhibition in patient 3 (right pallidal infarction), and subacute obsessivecompulsive disorders in patient 4 (right pallidal infarction). The symptoms were all present within a few seconds in patients 1,2 , and 3 , and occurred as a crescendo over 45 minutes in patient 4 (subacute obsessivecompulsive disorder). Neurocognitive disorders were acute verbal short term memory deficit in three patients (left lenticular lesion in patient 1 and right lenticular lesions in patients 3 and 4). There was no loss of consciousness, nor headache. The SPECT study showed a slight decrease of blood flow on the homolateral temporal area, despite the lack of cortical lesions on MRI.

Four patients had hypertension, one had diabetes, four were cigarette smokers, and two had hypercholesterolaemia. One patient had a history of myocardial ischaemia and one patient had cardiac arrhythmia. Three patients had lacunar infarction due to microangiopathy, and one had a cardiac embolism.

Follow up (six months to two years ; mean 18 months) data confirmed that the sequaelae were minor, with slight memory disorders in one patient. diagnosed from ECG recordings. Len lar infarction was distinguished from atherosclerosis in patients with vascular risk factors (hypertension, diabetes, hypercholesterolaemia, tobacco smoking). Embolism was diagnosed in patients with plaques or a stenosis within the appropriate carotid artery, shown on Doppler and angiography, or with cardiac embolic sources as well as arrhythmia, or ovale foramen
PUTAMINAL INFARCTS (FIG 2)

These were the most frequent type of infarct (16 patients). Table 2 shows the characteristics of these patients. There were 10 men and six women, mean age 73.5 years. There were nine

Table 1 Clinical disorders with pallidal infarct (four patients)

\begin{tabular}{|c|c|c|c|c|c|c|c|c|c|}
\hline Patient & $\begin{array}{l}\text { Age } \\
(y)\end{array}$ & Sex & $\begin{array}{l}\text { Side of } \\
\text { lesion }\end{array}$ & Onset & Headache & $\begin{array}{l}\text { Motor } \\
\text { palsy }\end{array}$ & $\begin{array}{l}\text { Neurocognitive } \\
\text { problems }\end{array}$ & $\begin{array}{l}\text { Behavioural } \\
\text { problems }\end{array}$ & Outcome \\
\hline 1 & 72 & M & Left & Acute & 0 & 0 & Memory & Abulia & Impaired memory \\
\hline 2 & 75 & $M$ & Left & Acute & 0 & 0 & 0 & Abulia & Normal \\
\hline 3 & 69 & $M$ & Right & Acute & 0 & 0 & Memory & Disinhibition & Normal \\
\hline 4 & 74 & $\mathrm{~F}$ & Right & Subacute & 0 & 0 & Memory & $\begin{array}{l}\text { Obsessive- } \\
\text { compulsive }\end{array}$ & Normal \\
\hline
\end{tabular}




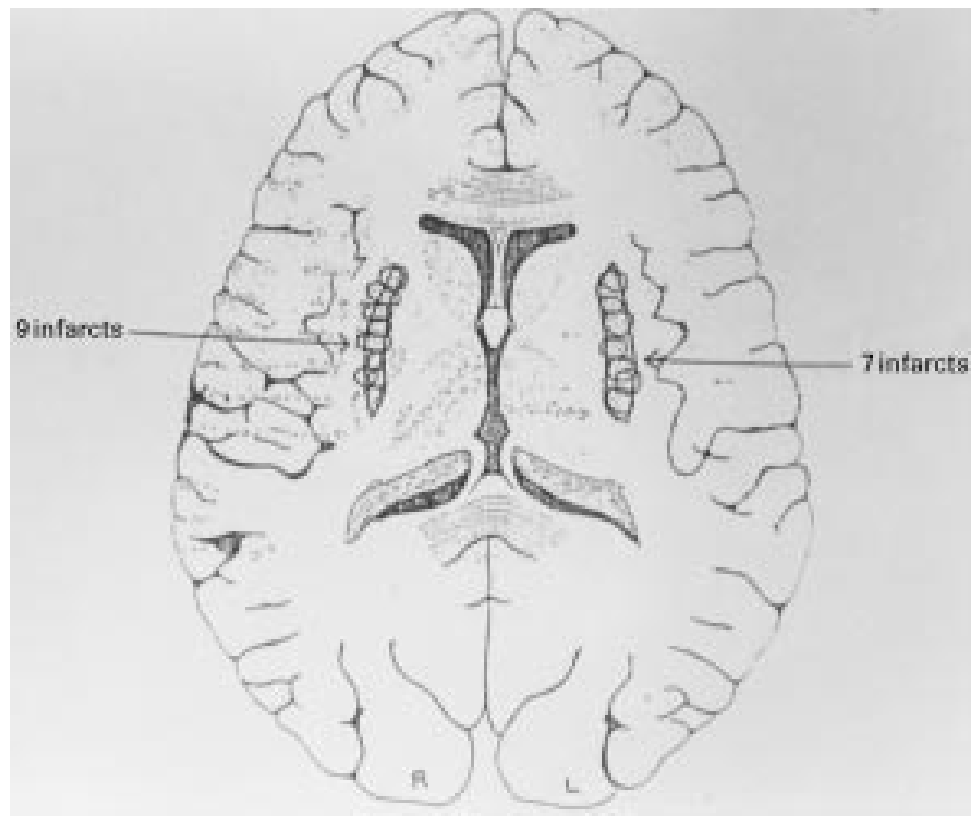

Figure 2 Schematic template showing lesion sites in the 16 patients with putaminal infarct. right infarcts and seven left infarcts. The main symptoms were acute neuropsychological and motor dysfunction. We noted an acute but regressive dysphasia characterised by hypophonia, verbal paraphasia, non-fluent speech with silence, loss of words without any impairment of comprehension, and preserved repetition in five right handed patients with left putaminal infarction (patients 5, 6, 8, 11, and 16). An acute short term memory deficit was seen in two patients with right putaminal infarction (patients 3 and 4) and in five patients with left putaminal infarction (patients 1, 8, 11, 13, and 16). Seven patients showed an acute but transient lower facial weakness only for automatic emotional movements with symmetric voluntary contraction (patients $1,3,5,8,10$, 14, and 16) and two had an acute hemineglect with right putaminal infarction (patients 2 and 7).

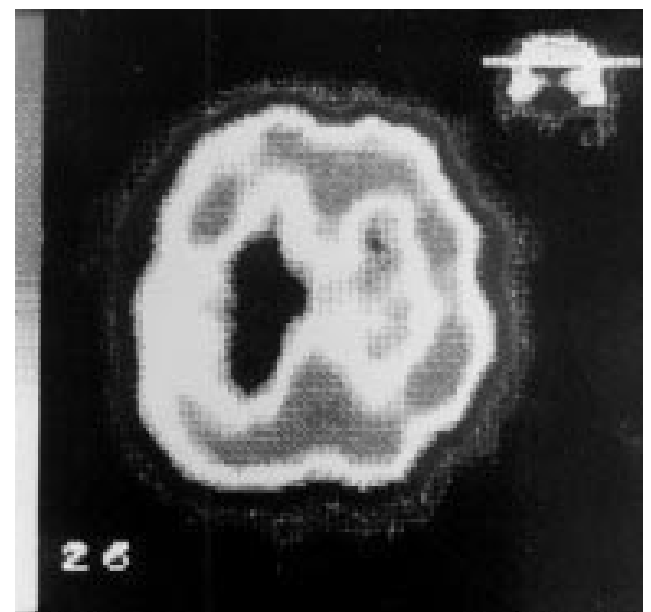

Figure 3 (patient 4): Right putaminal infarct: reduced right cortical frontotemporal blood flow on SPECT.

Acute but regressive chorea was present in patient 2 (right putaminal infarct) and in patient 6 (left putaminal infarct). Acute dystonia was seen in two patients with left putaminal infarction (patients 11 and 13) and in two patients with right putaminal infarction (patients 9 and 15). Acute cephalalgia was present in five patients $(1,5,8,10$, and 12).

The SPECT study showed a decrease of blood flow on the homolateral frontotemporal lobe despite the lack of cortical capsular lesions on MRI (fig 3).

Ten patients had hypertension, two patients had diabetes, eight were cigarette smokers, and seven had hypercholesterolaemia. Five patients had ischaemic heart disease, and three had a cardiac arrhythmia. A lacunar infarction was diagnosed in 10 patients, whereas a possible embolic cause was suspected in six patients. There were no deaths during follow up (eight months to 28 months; mean 22 months). Memory was impaired in seven patients $(1,3,4,8,11,13$, and 16), and there were language disturbances in two patients ( 5 and 6).

Table 2 Clinical disorders with putaminal infarct (16 cases)

\begin{tabular}{|c|c|c|c|c|c|c|c|c|c|}
\hline Patient & $\begin{array}{l}\text { Age } \\
(y)\end{array}$ & Sex & $\begin{array}{l}\text { Side of } \\
\text { lesion }\end{array}$ & Onset & Headache & Motor problems & $\begin{array}{l}\text { Neurocognitive } \\
\text { problems }\end{array}$ & $\begin{array}{l}\text { Behavioural } \\
\text { problems }\end{array}$ & Outcome \\
\hline 1 & 72 & M & Left & Acute & Yes & Facial palsy & Memory $\downarrow$ & 0 & Memory $\downarrow$ \\
\hline 2 & 75 & $M$ & Right & Acute & 0 & Chorea & Hemineglect & 0 & Normal \\
\hline 3 & 79 & $\mathrm{~F}$ & Right & Acute & 0 & Facial palsy & Memory $\downarrow$ & 0 & Memory $\downarrow$ \\
\hline 4 & 72 & $\mathrm{~F}$ & Right & Subacute & 0 & Hemiparkinsonism & Memory $\downarrow$ & 0 & Memory $\downarrow$ \\
\hline 5 & 69 & $M$ & Left & Acute & Yes & Facial palsy & Language & 0 & Language $\downarrow$ \\
\hline 6 & 77 & $M$ & Left & Acute & 0 & Chorea & Language & 0 & Language $\downarrow$ \\
\hline 7 & 73 & $M$ & Right & Subacute & 0 & Facial palsy & Hemineglect & 0 & Normal \\
\hline 8 & 74 & M & Left & Acute & Yes & Facial palsy & $\begin{array}{l}\text { Memory }+ \\
\text { language } \downarrow\end{array}$ & 0 & Memory $\downarrow$ \\
\hline 9 & 75 & $\mathrm{~F}$ & Right & Acute & 0 & Hemidystonia & 0 & 0 & Normal \\
\hline 10 & 72 & $\mathrm{~F}$ & Right & Acute & Yes & Facial palsy & 0 & 0 & Normal \\
\hline 11 & 68 & $M$ & Left & Acute & 0 & Hemidystonia & $\begin{array}{l}\text { Memory }+ \\
\text { language } \downarrow\end{array}$ & 0 & Memory $\downarrow$ \\
\hline 12 & 70 & $\mathrm{~F}$ & Right & Acute & Yes & 0 & 0 & 0 & Normal \\
\hline 13 & 71 & $M$ & Left & Subacute & 0 & Hemidystonia & Memory $\downarrow$ & 0 & Memory $\downarrow$ \\
\hline 14 & 84 & $M$ & Right & Acute & 0 & Facial palsy & & Confusion & Normal \\
\hline 15 & 72 & $M$ & Right & Acute & 0 & Hemidystonia & 0 & 0 & Normal \\
\hline 16 & 73 & $\mathrm{~F}$ & Left & Acute & 0 & Facial palsy & $\begin{array}{l}\text { Memory }+ \\
\text { language } \downarrow\end{array}$ & 0 & Memory $\downarrow$ \\
\hline
\end{tabular}




\section{Discussion}

This large clinical and radiological study of patients with lenticular infarcts attempted to classify the types of clinical syndromes, the causes of infarct, and their prognosis.

Our patients illustrate the complex functions of the lenticular nucleus. We distinguished, in agreement with Bhatia and Marsden, ${ }^{10}$ two major distinct clinical syndromes corresponding to the two main areas of the lenticular nucleus: the globus pallidus and the putaminal nucleus.

Lesions located to the lentiform nucleus often cause a special motor disorder and are often associated with a behavioural disorder unusual in other strokes.

Secondly, it is possible to distinguish acute and delayed disorders. The acute stage is usually spectacular. Dystonia is by far the commonest symptom in small and large lesions confined to the lentiform nucleus. Dystonia involved the upper limb in four patients without any complete hemiparesis. This picture is more often found with a contralateral capsulolenticular infarct. ${ }^{11-14}$ In the seven patients with a transient central facial palsy and a normal capsule on MRI, it is not possible to exclude a lesion of the adjacent internal capsule, but we think that this was unlikely.

Dystonia occurs in $63 \%$ of putaminal lesions and in $37 \%$ of globus pallidus lesions. ${ }^{13}$ Most lesions inducing dystonia are in the lentiform nucleus, mostly when the putamen is involved, as in our patients. An acute transient chorea was present in two patients without any lesion in the caudate nucleus, and an involvement of the fibres linking the lenticular and the caudate nuclei is possible. In patient 4 , a parkinsonism syndrome was present with akinesia and rigidity on the left side.

The behavioural disturbances were unexpected. Among them, abulia was common. The loss of mental and motor initiative and drive that define abulia, inducing apathy and blunting of responses, may be linked to damage of the caudate, but is related in our series to damage of the globus pallidus.

The neuropsychological dysfunctions are in general transient and slight: dysphasia is characterised by hypophonia, a reduced output, verbal paraphasias, and preserved repetition. These speech abnormalities seem to be a transcortical form of aphasia, sometimes associated with acalculia or apraxia. ${ }^{15-19}$ Other acute neuropsychological disturbances are found, such as acute short term memory dysfunction, and confusion, ${ }^{20}$ with lesions in the lower putaminal area. ${ }^{21}$ The short term memory disturbances may be induced by damage to the putaminal nucleus, as this nucleus is involved in the activation of the memory process? ${ }^{21}$ Hemineglect found in two patients is considered as an anosognosia and an asomatognosia with loss of awareness and paresis of the left hemibody. It is different from akinesia.
It is important to note that only one patient (12) had neither motor nor cognitive disorders, but an acute headache disclosed the lenticular stroke.

Later follow up studies showed that the prognosis was good. Motor and cognitive sequaelae were absent in three patients with pallidal infarcts and in seven patients with putaminal infarcts. The usual sequaela was cognitive with impaired memory, and occurred in one patient with a pallidal infarct and in seven patients with putaminal infarcts. It was mostly short term memory that was affected. Language disorders were present in two patients, with a slight loss of words. These cognitive disorders may be induced by subcortical lesions ${ }^{10}$ but the explanation for this phenomenon remains unclear. In seven of eight patients with behavioural changes and lesions in the lentiform nuclei, Laplane et $\mathrm{l}^{22}$ foundwith metabolic studies using ${ }^{18} \mathrm{~F}$-fluorodeoxyglucose-a significant cortical glucose hypometabolism in laterofrontal and mediofrontal regions, by a deafferentation process. The decrease of homolateral frontotemporal cerebral blood flow seen on SPECT in our patients with no lesion on MRI may explain the behavioural and neurocognitive changes by the presence of a diaschisis phenomenon ${ }^{22}$ between the lenticular nucleus and the homolateral frontotemporal cortex.

Lacunar infarction due to hypertensive or diabetic arteriolopathy has been considered the most common cause of lentiform infarction with emboligenic heart disease as the second cause.

In conclusion, it was possible to identify the clinical symptoms of a single lesion in the pallidus nucleus and in the putaminal nucleus, in which behavioural, cognitive, and movements disorders are important. After an acute and spectacular onset, outcome is in general excellent. A disease of the small arteries must be involved.

1 Tolosa ES, Santamania J. Parkinsonism and basal ganglia infarcts. Neurology 1984;34:1516-8.

2 Graff-Radford NR, Damasio H, Yamada T, Eslinger PJ, Damasio AR Non-haemorrhagic thalamic infarction. Brain 1985;108:485-516.

3 Orgogozzo JM, Bogouslavsky J. Lacunar syndromes. In: Toole JF, ed. Handbook of clinical neurology. Vol 10 (54): vasToole JF, ed. Handbook of clinical neurology. Vol 10 (54): vas-

4 Nelson HE. A modified card sorting test sensitive to frontal lobe defects. Cortex 1976;12:13-24.

5 Army individual test battery. Manuel of directions and scoring. Washington: War Department, Adjutant's General Office, Washington: War Department, Adjutant's General Office, 1944

6 Luria AR. Higher cortical functions in man. New York: Basic, 1966.

7 Osterrieth P. Le test de copie d'une figure complexe. Arch Psychol 1965;23:18-24.

8 Golden CJ. Stroop colour and word test. In: Golden CJ, ed. A manual for clinical and experimental uses. Chicago: Stoelting, 1978 .

9 Fisher CM. The arterial lesions underlying lacunes. Acta Neuropathol (Berl) 1969;12:1-15.

10 Bathia KP, Marsden CD. The behavioural and motor consequences of focal lesions of the basal ganglia in man. Brain 1994;117:859-76.

11 Russo LS Jr. 1 Focal dystonia and lacunar infarction of the basal ganglia. Arch Neurol 1983;40:61-2.

12 Grimes JD, Hassan MN, Guarrington AM, D'Alton J. Delayed onset post-hemiplegic dystonia: CT demonstration of basal ganglia pathology. Neurology 1982;32:1033- 
13 Marsden CD, Obeso JA, Zarranz JJ, Lang AE. The anatomical basis of symptomatic hemidystonia. Brain anatomical basis

14 Crossman AR, Sambrook MA, Jackson A. Experimental hemichorea/ hemiballismus in the monkey. Studies on the intracerebral site of action in a drug-induced dyskinesia Brain 1984;107:579-96.

15 Corbett AJ, Maccusker EA, Davidson OR. Acalculia following a dominant hemisphere subcortical infarct. Arch Neurol 1986;43:964-6.

16 De Renzi E, Faglioni P, Scarpa M, Grisi G. Limb apraxia in patients with damage confined to the left basal ganglia and thalamus. 7 Neurol Neurosurg Psychiatry 1986;49: 1030-8.

17 Fisher CM. Lacunar strokes and infarcts: a review. Neurology 1982;32:871-6.
18 Van Gijn J, Kraaijeveld CL. Blood pressure does not predict lacunar infarction. F Neurol Neurosurg Psychiatry 1982;45: 147-50.

9 Santamaria J, Graus F, Rubro F, Arbizu T, Peres J. Cerebral infarction of the basal ganglia due to embolism from the heart. Stroke 1983;14:911-4.

20 Fisher CM. Capsular infarcts: the underlying vascular lesions: Arch Neurol 1979;36:65-73.

21 Kashibara M, Matsumoto K. Acute capsular infarction. Location of the lesions and the clinical features. Neuroradiology 1985;27:248-53.

22 Laplane D, Levasseur M, Pillon B, Dubois B, Baulac M, Mazoyer B, et al. Obsessive-compulsive and other behavioural changes with bilateral basal ganglia lesions. Brain 1989;112:699-725. 